\title{
Correction to: Zafaria cholistanensis gen. nov. sp. nov., a moderately thermotolerant and halotolerant actinobacterium isolated from Cholistan desert soil of Pakistan
}

\author{
Arshia Amin 1,2,3 - Iftikhar Ahmed ${ }^{2}$ (D) Nauman Khalid ${ }^{4} \cdot$ Peter Schumann $^{5} \cdot$ Hans-Jürgen Busse $^{6} \cdot$ Inam Ullah Khan $^{7}$. \\ Ahmad Ali ${ }^{2}$. Shuai $\mathrm{Li}^{1} \cdot$ Wen-Jun $\mathrm{Li}^{1}$
}

Published online: 22 June 2021

(c) Springer-Verlag GmbH Germany, part of Springer Nature 2021

\section{Correction to: \\ Archives of Microbiology (2021) 203:1717-1729 \\ https://doi.org/10.1007/s00203-020-02176-4}

Subsequent to the publication of the above paper, it has been brought to our attention that in the original article, the protologue to describe the novel genus Zafaria and novel species Zafaria cholistanensis, were not according to the rules of the International Code of Nomenclature of Prokaryotes. According to Rule 15 of the ICNP, the nomenclatural type of a genus should be a species. However, a strain was assigned as the type. Therefore, as per requirement for validation of the names Zafaria and Zafaria cholistanensis the entire protologues of the genus and the species are reprinted with the necessary corrections:

Description of Zafaria gen. nov.

Zafaria (Za.fa'ri.a. N.L. fem. n. Zafaria, named in honor of Dr. Yusuf Zafar, a national distinguished scientist in the subject of microbiology and biotechnology in Pakistan).

Cells are Gram-staining positive and non-spore forming with rod to coccus life cycle. Positive for catalase but

The original article can be found online at https://doi.org/10.1007/ s00203-020-02176-4.

Iftikhar Ahmed

iftikhar.ahmed@parc.gov.pk

Wen-Jun Li

liwenjun3@mail.sysu.edu.cn

1 State Key Laboratory of Biocontrol, Guangdong Provincial Key Laboratory of Plant Resources and Southern Marine Science and Engineering Guangdong Laboratory (Zhuhai), School of Life Sciences, Sun Yat-Sen University, Guangzhou 510275, People's Republic of China

2 National Culture Collection of Pakistan (NCCP), Bio-Resources Conservation Institute (BCI), National Agricultural Research Centre (NARC), Park Road, Islamabad 45500, Pakistan negative for production of $\mathrm{H}_{2} \mathrm{~S}$, and $\beta$-galactosidase (ONPG) tests. The dominant respiratory lipoquinone system is MK$9\left(\mathrm{H}_{2}\right)$ along with MK-8( $\left.\mathrm{H}_{2}\right)$ as minor component. The major cellular fatty acid is anteiso- $\mathrm{C}_{15: 0}$. The polar lipids profile predominantly consists of diphosphatidylglycerol (DPG), phosphatidylglycerol (PG), glycolipid (GL). Cell-wall peptidoglycan is of the type A $3 \alpha$ L-Lys - L-Ala (A11.4). The type species is Zafaria cholistanensis. The genus belongs to the family Micrococcaceae, Order Actinomycetales.

Description of Zafaria cholistanensis gen. nov. sp. nov.

Zafaria cholistanensis (cho.lis.tan.en'sis. N.L. fem. adj. cholistanensis pertaining to the Cholistan Desert in Punjab, Pakistan, where the type strain was isolated).

In addition to the characteristics described for the genus, the species has the following features. Cells are Gram-staining positive and non-spore forming with rod to coccus life cycle. Cells are aerobic and non-motile. Colonies are round and off white in colour. The optimum temperature, $\mathrm{pH}$ and $\mathrm{NaCl}$ concentration for growth are $37{ }^{\circ} \mathrm{C}, 7.0-8.0$ and $2 \%$ $(\mathrm{w} / \mathrm{v})$, respectively. Positive for catalase but negative for production of $\mathrm{H}_{2} \mathrm{~S}$ and $\beta$-galactosidase (ONPG) tests. Positive for assimilation of D-sorbitol, $\mathrm{N}$-acetyl glucosamine and nitrate reduction. Positive for acid production from inulin

3 Department of Bioinformatics and Biosciences, Capital University of Science and Technology, Islamabad, Pakistan

4 School of Food and Agricultural Sciences, University of Management and Technology, Lahore 54000, Pakistan

5 Leibniz Institut DSMZ-Deutsche Sammlung, Von Mikroorganismen Und Zellkulturen $\mathrm{GmbH}$, Inhofenstraße 7B, 38124 Braunschweig, Germany

6 Institut Für Mikrobiologie, Veterinärmedizinische Universität Wien, Veterinärplatz 1, 1210 Wien, Austria

7 Institute of Microbiology, Faculty of Veterinary and Animal Sciences, Gomal University, Dera Ismail Khan, Pakistan 
and glycerol but negative from D-galactose, D-melezitose, D-raffinose, D-turanose, D-lactose, D-lyxose, D-fructose, L-rhamnose, D-melibiose and D-trehalose. Strong enzyme activity is observed for esterase lipase (C 8), alkaline phosphatase, leucine arylamidase, trypsin, acid phosphatase, naphthol-As-BI-phosphohydrolase, $\alpha$-glucosidase. Positive for utilization of substrates as carbon source: D-galactose, $\alpha$-hydroxy butyric acid, $\alpha$-ketoglutaric acid, malonic acid, L-asparagine, glycyl-L-glutamic acid, L-proline, L-pyroglutamic acid, L-serine and putrescine; weakly positive for D,L-lactic acid, succinamic acid, propionic acid, L-glutamic acid (Biolog GN III microplates ${ }^{\mathrm{TM}}$ ). Chemotaxonomic data of strain NCCP- $1664^{\mathrm{T}}$ showed peptidoglycan type as A3 $\alpha$ L-Lys-L-Ala (A11.4); menaquinones as MK-9 $\left(\mathrm{H}_{2}\right)(67 \%)$, MK- $8\left(\mathrm{H}_{2}\right)(32 \%)$ and MK-7 $\left(\mathrm{H}_{2}\right)(1 \%)$ and polar lipids profile comprising of diphosphatidylglycerol, phosphatidylglycerol, phosphatidylinositol, digalactosyldiacylglycerol, small amounts of monogalactosyldiacylglycerol, trimannosyldiacylglycerol and three unidentified lipids. The major wholecell sugars are galactose and glucose. Major fatty acids are

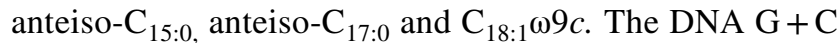
content is $70.0 \mathrm{~mol} \%$.

The type strain is NCCP- $1664^{\mathrm{T}}\left(=\mathrm{DSM} 29936^{\mathrm{T}}=\mathrm{KCTC}\right.$ $39549^{\mathrm{T}}$ ), which was isolated from soil sample collected from Cholistan desert, Bahawalpur, Pakistan. The DDBJ/EMBL/ GenBank accession number for the 16S rRNA gene sequence of Zafaria cholistanensis NCCP-1664 ${ }^{\mathrm{T}}$ is LC065376. The bio-project, bio-sample and whole-genome sequences of strain NCCP-1664 ${ }^{\mathrm{T}}$ have been deposited to DDBJ/EMBL/ GenBank under the accession numbers PRJDB8509, SAMD00178857 and BKDJ01000000, respectively.

The authors apologize for this error and state that this does not change the scientific conclusions of the article in any way.

Publisher's Note Springer Nature remains neutral with regard to jurisdictional claims in published maps and institutional affiliations. 